\title{
A Survey of Some Recent Results on Nonlinear Fault Tolerant Control
}

\author{
Mouhacine Benosman \\ Temasek Laboratories, National University of Singapore, 5A, Engineering Drive 1, 9-02, Singapore 117411 \\ Correspondence should be addressed to Mouhacine Benosman, tslmb@nus.edu.sg \\ Received 19 June 2009; Accepted 7 September 2009 \\ Academic Editor: Jose Balthazar \\ Copyright (C) 2010 Mouhacine Benosman. This is an open access article distributed under the \\ Creative Commons Attribution License, which permits unrestricted use, distribution, and \\ reproduction in any medium, provided the original work is properly cited. \\ Fault tolerant control (FTC) is the branch of control theory, dealing with the control of systems \\ that become faulty during their operating life. Following the systems classification, as linear and \\ nonlinear models, FTC can be classified in two different groups, linear FTC (LFTC) dealing with \\ linear models, and the one of interest to us in this paper, nonlinear FTC (NFTC), which deals with \\ nonlinear models. We present in this paper a survey of some of the results obtained in these last \\ years on NFTC.
}

\section{Introduction}

Due to the complexity of modern engineering systems, it is increasingly important to ensure their reliability. This has motivated researchers to concentrate on FTC, which is primarily meant to ensure safety, that is, the stability of a system after the occurrence of a fault in the system. There are two approaches to synthesize controllers that are tolerant to system faults. One approach, known as passive FTC, aims at designing a controller which is a priori robust to some given expected faults. Another approach, known as active FTC, relies on the availability of a fault detection and diagnosis (FDD) block that gives, in real-time, information about the nature and the intensity of the fault. This information is then used by a control reconfiguration block to adjust online the control effort in such a way to maintain stability and to optimize the performance of the faulty system.

Passive FTC has the drawback to be reliable only for the class of faults expected and taken into account in the design of the passive FTC. Furthermore, the performances of the closed-loop are not optimized for each fault scenario. However, it has the advantage to avoid the time delay due to online diagnosis of the faults and reconfiguration of the controller, required in active FTC $[1,2]$, which is very important in practical situations where the time windows during which the system stays stabilizable is very short, for example, the unstable 
double inverted pendulum example [3]. In practical applications passive FTCs complement active FTC schemes. Indeed, passive FTCs are necessary during the fault detection and estimation phases [4], where passive FTCs are used to ensure the stability of the faulty system, before switching to active FTCs, that recover some performance after the fault is detected and estimated. Another scenario where passive FTC is used as a complement of active FTC is in the switching-based active FTC, where the active FTC switches between different passive FTC, each controller being designed off-line to cope with a finite number of expected faults and stored in a controller bank; see for example, [5]. Several passive FTC methods have been proposed, mainly based on robust theory, for example, multiobjective linear optimization and LMIs techniques [6], QFT method [7, 8], $H_{\infty}[3,9]$, absolute stability theory [10], nonlinear regulation theory [11, 12], Lyapunov reconstruction [13, 14], and passivity-based FTC [15]. As for active FTC, many methods have been proposed for active LFTC, for example, [16-21], as well as for NFTC, for example, [14, 22-35]. As said before, this paper aim is to present some of the recent results on NFTC thus we will not further present LFTC here, and we refer the reader to other survey papers for linear systems; see for example, [36-38].

In $[11,12]$ the nonlinear regulation theory was used to solve the NFTC problem for particular practical examples, that is, robot manipulators in [12] and induction motors in [11]. The faults treated were modelled as additive actuator faults. In [13, 39] Lyapunov reconstruction techniques were used to solve the problem of loss of actuator effectiveness for nonlinear models affine in the control. The main drawback of this scheme is that it is based on the apriori knowledge of a stabilizing feedback for the nominal safe model and the knowledge of the associated Lyapunov function in closed form. Furthermore, the problem of inputs saturation has not been solved in this work. In [15] the authors studied the case of nonlinear systems with multiplicative actuator faults, and considered the case of systems with inputs saturation. In active NFTC field we also quote [24], where the authors studied the NFTC problem for a particular class of continuous nonlinear models, that is, linear in the control, and proposed a new adaptive fault estimation module, complemented with a control reconfiguration block. In [14], the authors study a specific problem of active FTC, namely, the problem of graceful performance degradation. This problem aims to define online new performances for the faulty system, these performances having to be feasible by the faulty system within its states/actuators limits. Indeed, after the occurrence of a fault the faulty system is expected to be unable to perform the tasks required and planned initially for the safe system. Therefore, new tasks, less demanding, have to be generated online for the faulty system. The idea used there is based on two main stages. The first stage concerns online trajectory planning or reshaping, using online optimization scheme that generates online the closest trajectory to the nominal one, but without violating the new constraints of the faulty system. The second stage concerns the control reallocation problem, using nonlinear model predictive control (NMPC). This scheme deals with nonminimum phase nonlinear models affine in the control. We also refer to $[28,29]$ where uncertain nonlinear models with constrained inputs, were considered.

An important part of FTC is the one specializing in actuator faults. Indeed, FTCs dealing with actuator faults are relevant for practical application and have already been the subject of many publications $[13,15,18,19,25,33,39-49]$. The nonlinear case has been studied in [25], where active FTC with respect to additive actuator faults was studied for nonlinear systems affine in the control. Constrained actuators were considered, and statefeedback as well as output-feedback FDDs/FTCs were proposed. In [4, 50], an active NFTC has been proposed for the class of SISO nonlinear systems, with incipient faults. The structure 
of the FTC was based on three controllers: a nominal controller for the safe system, that guarantees the system trajectories boundedness until the fault is detected. Then, the NFTC was reconfigured to the second controller that recovers some control performances before the fault is isolated. After the isolation of the fault, a third controller was used based on the faulty model, to improve the control performances. The reconfiguration of the controllers was based on adaptive backstepping approaches. In [14, 33, 48] the authors used model predictive controllers (linear case in [48], and nonlinear case [14, 33]) to reconfigure the controller online after the isolation and estimation of the fault. Finally, we quote [51], where a class of delayed nonlinear systems, modelled with linear terms added to Lipschitz-like nonlinearities with delay terms have been studied. The authors proposed an adaptive LMIbased active NFTC to ensure the stability of the faulty model as well as some optimal performances.

We do not pretend here to present in details all the work quoted above, instead, we will concentrate on some of these results and point out pros and cons of each scheme. We also underline, that we will not report the proofs of the results here, the reader will be refereed to the corresponding paper for the detailed proofs.

This paper is structured as follows. In Section 2, we introduce some notations, and recall some definitions that will be used throughout the paper. Section 3, concerns passive NFTC, followed by active NFTC in Section 4. Finally, we conclude the paper in Section 5, by pointing out some open problems in NFTC.

\section{Preliminaries}

Throughout the paper we will use the $L_{2}$ norm denoted by $|\cdot|$, that is, for $x \in \mathbb{R}^{n}$ we define $|x|=\sqrt{x^{T} x}$. The notation $L_{f} h$ denotes the standard Lie derivative of a scalar function $h(\cdot)$ along a vector function $f(\cdot)$. We also denote by $\tanh (\cdot)$ the hyperbolic tangent function and by $h^{(l)}$ the $l$ th-order-derivative of the scalar function $h$. Let us introduce now some definitions from [52], that will be frequently used in the sequel.

Definition 2.1 (see [52, page 45]). The solution $x\left(t, x_{0}\right)$ of the system $\dot{x}=f(x), x \in \mathbb{R}^{n}, f$ locally Lipschitz, is stable conditionally to $Z$, if $x_{0} \in Z$, and for each $\epsilon>0$ there exists $\delta(\epsilon)>0$ such that

$$
\left|\tilde{x}_{0}-x_{0}\right|<\delta, \quad \tilde{x}_{0} \in Z \Longrightarrow\left|x\left(t, \tilde{x}_{0}\right)-x\left(t, x_{0}\right)\right|<\epsilon, \quad \forall t \geq 0
$$

If, furthermore, there exist $r\left(x_{0}\right)>0$, s.t. $\left|x\left(t, \tilde{x}_{0}\right)-x\left(t, x_{0}\right)\right| \Rightarrow 0$, for all $\left|\tilde{x}_{0}-x_{0}\right|<$ $r\left(x_{0}\right)$ and $\tilde{x}_{0} \in Z$, the solution is asymptotically stable conditionally to $Z$. If $r\left(x_{0}\right) \rightarrow \infty$, the stability is global.

Definition 2.2 (see [52, page 48]). Consider the system $H: \dot{x}=f(x, u), y=h(x, u), x \in \mathbb{R}^{n}$, $u, y \in \mathbb{R}^{m}$, with zero inputs, that is, $\dot{x}=f(x, 0), y=h(x, 0)$, and let $Z \subset \mathbb{R}^{n}$ be its largest positively invariant set contained in $\left\{x \in \mathbb{R}^{n} \mid y=h(x, 0)=0\right\}$. We say that $H$ is globally zero-state detectable (GZSD) if $x=0$ is globally asymptotically stable conditionally to $Z$. If $\mathrm{Z}=\{0\}$, the system $H$ is zero-state observable (ZSO). 
Definition 2.3 (see [52, page 27]). We say that $H$ is dissipative in $X \subset \mathbb{R}^{n}$ containing $x=0$, if there exists a function $S(x), S(0)=0$ such that for all $x \in X$

$$
S(x) \geq 0, \quad S(x(T))-S(x(0)) \leq \int_{0}^{T} \omega(u(t), y(t)) d t
$$

for all $u \in U \subset \mathbb{R}^{m}$ and all $T>0$ such that $x(t) \in X$, for all $t \in[0, T]$, where the function $\omega: \mathbb{R}^{m} \times \mathbb{R}^{m} \rightarrow \mathbb{R}$, called the supply rate, is locally integrable for every $u \in U$, that is, $\int_{t_{0}}^{t_{1}}|\omega(u(t), y(t))| d t<\infty, \forall t_{0} \leq t_{1}$. $S$ is called the storage function. If the storage function is differentiable, the previous conditions write as

$$
\dot{S}(x(t)) \leq \omega(u(t), y(t)) .
$$

The system $H$ is said to be passive if it is dissipative with the supply rate $w(u, y)=u^{T} y$.

Remark 2.4. The definitions of (ZSD) and (ZSO) are simply an extension to the nonlinear case of the classical notions of detectability and observability for linear systems; see for example, [53]. Section 3 .

We will also need the following definition to study the case of time-varying faults in

Definition 2.5 (see [54]). A function $\bar{x}:[0, \infty) \rightarrow \mathbb{R}^{n}$ is called a limiting solution of the system $\dot{x}=f(t, x)$, and $f$ a smooth vector function, with respect to an unbounded sequence $t_{n}$ in $[0, \infty)$, if there exist a compact $\mathcal{\kappa} \subset \mathbb{R}^{n}$ and a sequence $\left\{x_{n}:\left[t_{n}, \infty\right) \rightarrow \mathcal{\kappa}\right\}$ of solutions of the system such that the associated sequence $\left\{\widehat{x}_{n}: \rightarrow x_{n}\left(t+t_{n}\right)\right\}$ converges uniformly to $\bar{x}$ on every compact subset of $[0, \infty)$.

Definition 2.6 (see [55, page 144]). A continuous function $\alpha:[0, a) \rightarrow[0, \infty)$ is said to belong to class $\mathcal{K}$ if it is strictly increasing and $\alpha(0)=0$. A continuous function $\beta:[0, a) \times$ $[0, \infty) \rightarrow[0, \infty)$ is said to belong to class $\mathcal{L} \perp$ if for each fixed $s$ the mapping $\beta(r, s)$ belongs to class $\mathcal{K}$ with respect to $r$ and for each fixed $r$ the mapping $\beta(r, s)$ is decreasing with respect to $s$ and $\beta(r, s) \rightarrow 0$ as $s \rightarrow \infty$.

Definition 2.7. A system is said of nonminimum phase, if it has internal dynamics, and their associated zero dynamics are unstable in the Lyapunov sense.

Also, throughout this paper it is said that a statement $P(t)$ holds a.e. if the Lebesgue measure of the set $\{t \in[0, \infty) \mid P(t)$ is false $\}$ is zero [54]. We also mean by semiglobal stability of the equilibrium point $x^{0}$ for the autonomous system $\dot{x}=f(x), x \in \mathbb{R}^{n}$ with $f$ a smooth function, that for each compact set $K \subset \mathbb{R}^{n}$ containing $x^{0}$, there exist a locally Lipschitz state feedback, such that $x^{0}$ is asymptotically stable, with a basin of attraction containing $K$ (see [56, Definition 3, page 1445]). 


\section{Passive NFTC}

Let us start first with some passive NFTC algorithms. As we said before, these types of FTCs are not expected to "do all the job alone", since in practice they have to be associated with some active FTCs to obtain an efficient controller tolerant to faults.

\subsection{Lyapunov-Reconstruction-Based Passive NFTC}

We first consider nonlinear systems of the form

$$
\dot{x}=f(x)+g(x) u,
$$

where $x \in \mathbb{R}^{n}$ and $u \in \mathbb{R}^{m}$ represent, respectively, the state and the input vectors. The vector fields $f$, columns of $g$ are supposed to satisfy the classical smoothness assumptions, with $f(0)=0$. We also assume the system (3.1), locally reachable (in the sense of [57, Definition 5, page 400]). Adding to the previous classical assumptions, we need also the following to hold.

Assumption 3.1. We assume the existence of a nominal closed-loop control $u_{\text {nom }}(t, x)$, such that the solutions of the closed-loop system

$$
\dot{x}=f(x)+g(x) u_{\text {nom }}(t, x)
$$

satisfy $|x(t)| \leq \beta\left(\left|x\left(t_{0}\right)\right|, t-t_{0}\right), \forall x_{t_{0}} \in D, \forall t \geq t_{0}$, where $D=\left\{x \in \mathbb{R}^{n}|| x \mid<r_{0}\right\}, r_{0}>0$ and $\beta$ is $a$ class $\mathcal{\perp} \perp$ function.

Assumption 3.2. We assume here two types of actuator faults.

(i) Firstly, one considers faults that enter the system in an additive way; that is, the faulty model writes as

$$
\dot{x}=f(x)+g(x)(u+F(t, x))
$$

where $F$ represents the actuator fault and s.t. $|F(t, x)| \leq b(t, x)$, where $b:[0, \infty) \times D \rightarrow \mathbb{R}$ is $a$ nonnegative continuous function.

(ii) Secondly, one considers loss of actuator effectiveness, represented by a multiplicative matrix $\alpha$ as

$$
\dot{x}=f(x)+g(x) \alpha u,
$$

where $\alpha \in \mathbb{R}^{m \times m}$ is a diagonal continuous time variant matrix, with the diagonal elements $\alpha_{i i}(t), i=$ $1, \ldots, m$ s.t. $0<\epsilon_{1} \leq \alpha_{i i}(t) \leq 1$. 
The authors in [39] proved the following propositions.

Proposition 3.3. The control law

$$
u(t, x)=u_{n o m}(t, x)-\operatorname{sgn}\left(\left(\frac{\partial V}{\partial x} g\right)^{T}\right)(b(t, x)+\epsilon), \quad \epsilon>0
$$

where $u_{n o m}(t, x)$ is s.t. Assumption 3.1 is satisfied, $V$ is the associated Lyapunov function, $b(t, x)$ is defined in Assumption 3.2, and $\operatorname{sgn}(v)$ denotes the vector $\operatorname{sign}$ function, s.t. $\operatorname{sgn}(v)(i)=\operatorname{sgn}(v(i))$; ensures that the equilibrium point $x=0$ is locally UAS in D for the closed-loop system (3.3) and (3.5).

Proposition 3.4. The control law

$$
u(t, x)=u_{n o m}(t, x)-\operatorname{sgn}\left(\left(\frac{\partial V}{\partial x} g\right)^{T}\right)\left(\left|u_{n o m}\right|+\frac{\left|u_{n o m}\right|}{\epsilon_{1}} \beta_{1}\right), \quad \beta_{1} \geq 1
$$

where $u_{n o m}(t, x)$ s.t. Assumption 3.1 is satisfied, $V$ is the associated Lyapunov function, and $\operatorname{sgn}(\cdot)$ denotes the sign function; ensures that the equilibrium point $x=0$ is locally UAS in $D$ for the closedloop system (3.4) and (3.6).

These two controllers ensure robust stabilization with respect to additive as well as multiplicative actuators' faults; however, they are discontinuous; that is, due to the sign function, therefore the authors in [39] proposed the following two "continuous" versions of the previous propositions.

Proposition 3.5. The control law

$$
u(t, x)=u_{n o m}(t, x)-s a t\left(\left(\frac{\partial V}{\partial x} g\right)^{T}\right)(b(t, x)+\epsilon), \quad \epsilon>0
$$

ensures that the solutions of the closed-loop system (3.3) and (3.7) satisfy

$$
\begin{aligned}
& \forall x\left(t_{0}\right) \quad \text { s.t. }\left|x\left(t_{0}\right)\right| \leq \alpha_{2}^{-1}\left(\alpha_{1}\left(r_{0}\right)\right), \\
& \exists T \geq 0, \quad \text { s.t. } \begin{cases}|x(t)| \leq \beta\left(\left|x\left(t_{0}\right)\right|, t-t_{0}\right), & \forall t_{0} \leq t \leq t_{0}+T, \\
|x(t)| \leq \alpha_{2}^{-1}\left(\alpha_{1}(\tilde{x})\right), & \forall t \geq t_{0}+T,\end{cases}
\end{aligned}
$$


where, for a vector $v$,

$$
\begin{gathered}
\operatorname{sat}(v)= \begin{cases}\frac{v(i)}{\widetilde{\epsilon}}, & \text { if }|v(i)| \leq \widetilde{\epsilon}, \\
\operatorname{sgn}(v(i)), & \text { if }|v(i)|>\widetilde{\epsilon},\end{cases} \\
\tilde{x}=\alpha_{3}^{-1}\left(2 m \tilde{\epsilon} b_{\max }\right) \leq \alpha_{2}^{-1}\left(\alpha_{1}\left(r_{0}\right)\right), \\
b(t, x) \leq b_{\max }, \quad \forall t, \forall x \in D,
\end{gathered}
$$

and $\alpha_{1}, \alpha_{2}$, and $\alpha_{3}$ are class $\mathcal{K}$ functions in $D$ and $\beta$ is class $\mathcal{\mathcal { L }} \perp$.

Proposition 3.6. The control law

$$
u(t, x)=u_{\text {nom }}(t, x)-\operatorname{sat}\left(\left(\frac{\partial V}{\partial x} g\right)^{T}\right)\left(\left|u_{\text {nom }}\right|+\frac{\left|u_{\text {nom }}\right|}{\epsilon_{1}} \beta_{1}\right), \quad \beta_{1} \geq 1
$$

ensures that the solutions of the closed-loop system (3.4) and (3.10) satisfy

$$
\begin{aligned}
& \forall x\left(t_{0}\right), \text { s.t. }\left|x\left(t_{0}\right)\right| \leq \alpha_{2}^{-1}\left(\alpha_{1}\left(r_{0}\right)\right), \\
& \exists T \geq 0, \quad \text { s.t. } \begin{cases}|x(t)| \leq \beta\left(\left|x\left(t_{0}\right)\right|, t-t_{0}\right), & \forall t_{0} \leq t \leq t_{0}+T, \\
|x(t)| \leq \alpha_{2}^{-1}\left(\alpha_{1}(\tilde{x})\right), & \forall t \geq t_{0}+T,\end{cases}
\end{aligned}
$$

where, for a vector $v$,

$$
\begin{gathered}
\operatorname{sat}(v)= \begin{cases}\frac{v(i)}{\widetilde{\epsilon}}, & \text { if }|v(i)| \leq \widetilde{\epsilon}, \\
\operatorname{sgn}(v(i)), & \text { if }|v(i)|>\widetilde{\epsilon},\end{cases} \\
\tilde{x}=\alpha_{3}^{-1}\left(2 m \tilde{\epsilon} u_{\text {nom-max }}\right) \leq \alpha_{2}^{-1}\left(\alpha_{1}\left(r_{0}\right)\right), \\
\left|u_{\text {nom }}\right| \leq u_{\text {nom-max }}, \quad \forall t,
\end{gathered}
$$

and $\alpha_{1}, \alpha_{2}$, and $\alpha_{3}$ are class $\mathcal{K}$ functions in $D$ and $\beta$ is class $\mathcal{K} \perp$.

The two continuous controllers (3.7) and (3.10) and do not guarantee the local UAS anymore. However, they guarantee that the closed-loop trajectories are bounded by a class $\mathcal{K}$ function, and that this bound can be made as small as desired by choosing a small $\tilde{\epsilon}$ in the definition of the function sat. The passive NFTC recalled above is in closed form and thus easy to implement. However, they have two main drawbacks. Firstly, they are based on 
the availability of the closed-from expression of the Lyapunov function associated with the nominal stabilizing law, and secondly, they do not consider input saturations in the control design. Therefore, trying to overcome these limitations, other controllers have been proposed and are recalled hereinafter.

\subsection{Passivity-Based NFTC}

In [15], the passivity theory has been used to develop some new NFTC dealing with actuator multiplicative faults. These results are reported hereinafter.

Theorem 3.7. Consider the closed-loop system that consists of the faulty system (3.4), with constant unknown matrix $\alpha$, and the dynamic state feedback:

$$
\begin{gathered}
\dot{u}=-L_{g} W(x)^{T}-k \xi, \quad u(0)=0, \\
\dot{\xi}=\epsilon_{1}\left(-\left(L_{g} W(x)\right)^{T}-k \xi\right), \quad \xi(0)=0,
\end{gathered}
$$

where $W$ is a $C^{1}$ radially unbounded, positive semidefinite function, s.t. $L_{f} W \leq 0$, and $k>0$. Consider the fictitious system

$$
\begin{gathered}
\dot{x}=f(x)+g(x) \xi, \\
\dot{\xi}=\epsilon_{1}\left(-\left(L_{g} W\right)^{T}+\widetilde{v}\right), \\
y=h(\xi)=\xi .
\end{gathered}
$$

If the system (3.14) is (G)ZSD with the input $\widetilde{v}$ and the output $y$, then the closed-loop system (3.4) with (3.13) admits the origin $(x, \xi)=(0,0)$ as (globally) asymptotically stable $((G) A S)$ equilibrium point.

In Theorem 3.7, one of the necessary conditions is the existence of $W \geq 0$, s.t. the uncontrolled part of (3.3) satisfies $L_{f} W \leq 0$. To avoid this condition that may not be satisfied for some practical systems, the authors proposed the following Theorem.

Theorem 3.8. Consider the closed-loop system that consists of the faulty system (3.4), with constant unknown matrix $\alpha$, and the dynamic state feedback:

$$
\begin{gathered}
\dot{u}=\frac{1}{\epsilon_{1}}\left(-k(\xi-\beta K(x))-\beta L_{g} W^{T}+\beta \frac{\partial K}{\partial x}(f+g \xi)\right), \\
\beta=\operatorname{diag}\left(\beta_{11}, \ldots, \beta_{m m}\right), \quad 0<\frac{\tilde{\epsilon}_{1}}{\epsilon_{1}} \leq \beta_{i i} \leq 1, \\
\dot{\xi}=-k(\xi-\beta K(x))-\beta L_{g} W^{T}+\beta \frac{\partial K}{\partial x}(f+g \xi), \quad \xi(0)=0, \quad u(0)=0,
\end{gathered}
$$


where $k>0$ and the $C^{1}$ function $K(x)$ s.t. a $C^{1}$ radially unbounded, positive semidefinite function $W$ satisfying

$$
\frac{\partial W}{\partial x}(f(x)+g(x) \beta K(x)) \leq 0, \quad \forall x \in \mathbb{R}^{n}, \quad \forall \beta=\operatorname{diag}\left(\beta_{11}, \ldots, \beta_{m m}\right), \quad 0<\widetilde{\epsilon}_{1} \leq \beta_{i i} \leq 1 .
$$

Consider the fictitious system

$$
\begin{gathered}
\dot{x}=f(x)+g(x) \xi, \\
\dot{\xi}=\beta \frac{\partial K}{\partial x}(f+g \xi)-\beta L_{g} W^{T}+\widetilde{\widetilde{v}} \\
\tilde{y}=\xi-\beta K(x) .
\end{gathered}
$$

If (3.17) is (G)ZSD with the input $\widetilde{\tilde{v}}$ and the output $\tilde{y}$, for for all $\beta$ s.t. $\beta_{i i}, i=1, \ldots, m, 0<\widetilde{\epsilon}_{1} \leq$ $\beta_{i i} \leq 1$. Then, the closed-loop system (3.4) with (3.15) admits the origin $(x, \xi)=(0,0)$ as $(G) A S$ equilibrium point.

The previous theorems may guaranty global AS. However, the conditions required may be difficult to satisfy for some systems. Thus, the authors in [15] introduced the following control law that ensures, under less demanding conditions, semiglobal stability instead of global stability.

Theorem 3.9. Consider the closed-loop system that consists of the faulty system (3.4), with constant matrix $\alpha$, and the dynamic state feedback:

$$
\begin{gathered}
\dot{u}=-k\left(\xi-u_{\text {nom }}(x)\right), \quad k>0, \\
\dot{\xi}=-k \epsilon_{1}\left(\xi-u_{\text {nom }}(x)\right), \quad \xi(0)=0, \quad u(0)=0,
\end{gathered}
$$

where the nominal controller $u_{\text {nom }}(x)$ achieves semiglobal asymptotic and local exponential stability of $x=0$ for the safe system (3.1). Then, the closed-loop (3.4) with (3.18) admits the origin $(x, \xi)=(0,0)$ as semiglobal AS equilibrium point.

In [15], the practical problem of input saturation has been studied, and the following result on general nonlinear models, nonnecessarily affine on $u$, has been proposed.

Theorem 3.10. Consider the closed-loop system that consists of the faulty system:

$$
\dot{x}=f(x)+g(x, \alpha u) \alpha u
$$

for $\alpha \in\left[\epsilon_{1}, 1\right]$, and the static state feedback: 


$$
\begin{gathered}
u(x)=-\lambda(x) G(x, 0)^{T}, \\
G(x, 0)=\frac{\partial W(x)}{\partial x} \epsilon_{1} g(x, 0), \\
\lambda(x)=\frac{2 \bar{u}}{\left(1+\gamma_{1}\left(|x|^{2}+4 \bar{u}^{2}|G(x, 0)|^{2}\right)\right)\left(1+|G(x, 0)|^{2}\right)}>0, \\
\gamma_{1}=\int_{0}^{2 s} \frac{\bar{\gamma}_{1}(s)}{1+\bar{\gamma}_{1}(1)} d s, \\
\bar{\gamma}_{1}(s)=\frac{1}{s} \int_{s}^{2 s}\left(\tilde{\gamma}_{1}(t)-1\right) d t+s, \\
\tilde{\gamma}_{1}(s)=\max _{\left\{(x, u)|x|^{2}+|u|^{2} \leq s\right\}}\left\{1+\int_{0}^{1} \frac{\partial W(x)}{\partial x} \frac{\partial g\left(x, \tau \epsilon_{1} u\right)}{\partial u} d \tau\right\},
\end{gathered}
$$

where $W$ is a $C^{2}$ radially unbounded, positive semidefinite function, s.t. $L_{f} W \leq 0$. Consider the fictitious system:

$$
\begin{gathered}
\dot{x}=f(x)+g\left(x, \epsilon_{1} u\right) \epsilon_{1} u, \\
y=\frac{\partial W(x)}{\partial x} \epsilon_{1} g\left(x, \epsilon_{1} u\right) .
\end{gathered}
$$

If (3.21) is (G)ZSD, then the closed-loop system (3.19) with (3.20) admits the origin as (G)AS equilibrium point. Furthermore $|u(x)| \leq \bar{u}$, for all $x$.

For the particular case of affine nonlinear systems, that is, $g(x, u)=g(x)$, we have the following proposition, which is a direct consequence of Theorem 3.10.

Proposition 3.11. Consider the closed-loop system that consists of the faulty system (3.4), with constant unknown matrix $\alpha$, and the static state feedback:

$$
\begin{gathered}
u(x)=-\lambda(x) G(x)^{T}, \\
G(x)=\frac{\partial W(x)}{\partial x} \epsilon_{1} g(x), \\
\lambda(x)=\frac{2 \bar{u}}{1+|G(x)|^{2}} .
\end{gathered}
$$


where $W$ is a $C^{2}$ radially unbounded, positive semidefinite function, s.t. $L_{f} W \leq 0$. Consider the fictitious system:

$$
\begin{aligned}
& \dot{x}=f(x)+g(x) \epsilon_{1} u, \\
& y=\frac{\partial W(x)}{\partial x} \epsilon_{1} g(x) .
\end{aligned}
$$

If (3.23) is (G)ZSD, then the closed-loop system (3.4) with (3.22) admits the origin as (G)AS equilibrium point. Furthermore $|u(x)| \leq \bar{u}, \forall x$.

The time-varying versions, that is, for time-varying faults, of the previous results have also been proven in [15] and are recalled hereinafter.

Theorem 3.12. Consider the closed-loop system that consists of the faulty system (3.4) with the dynamic state feedback:

$$
\begin{array}{ll}
\dot{u}=-L_{g} W(x)^{T}-k \xi, \quad k>0, & u(0)=0 \\
\dot{\xi}=\tilde{\alpha}(t)\left(-\left(L_{g} W(x)\right)^{T}-k \xi\right), & \xi(0)=0,
\end{array}
$$

where $\widetilde{\alpha}(t)$ is a $C^{1}$ function, s.t. $0<\epsilon_{1} \leq \widetilde{\alpha}(t) \leq 1, \forall t$, and $W$ is a $C^{1}$, positive semidefinite function, such that

(1) $L_{f} W \leq 0$;

(2) the system $\dot{x}=f(x)$ is AS conditionally to the set $M=\{x \mid W(x)=0\}$;

(3) for all $(\bar{x}, \bar{\xi})$ limiting solutions for the system

$$
\begin{gathered}
\dot{x}=f(x)+g(x) \xi, \\
\dot{\xi}=\alpha(t)\left(-\left(L_{g} W\right)^{T}-k \xi\right), \\
y=h(x, \xi)=\xi,
\end{gathered}
$$

with respect to unbounded sequence $\left\{t_{n}\right\}$ in $[0, \infty)$, then if $h(\bar{x}, \bar{\xi})=0$, a.e., then either $(\bar{x}, \bar{\xi})\left(t_{0}\right)=(0,0)$ for some $t_{0} \geq 0$ or $(0,0)$ is a w-limit point of $(\bar{x}, \bar{\xi})$, that is, $\lim _{t \rightarrow \infty}(\bar{x}, \bar{\xi})(t) \rightarrow(0,0)$.

Then the closed-loop system (3.4) with (3.24) admits the origin $(x, \xi)=(0,0)$ as UAS equilibrium point.

Theorem 3.13. Consider the closed-loop system that consists of the faulty system:

$$
\dot{x}=f(x)+g(x, \alpha(t) u) \alpha(t) u,
$$


for $\alpha \in\left[\epsilon_{1}, 1\right], \forall t$, with the static state feedback:

$$
\begin{gathered}
u(x)=-\lambda(x) G(x, 0)^{T}, \\
G(x, 0)=\frac{\partial W(x)}{\partial x} g(x, 0), \\
\lambda(x)=\frac{2 \bar{u}}{\left(1+\gamma_{1}\left(|x|^{2}+4 \bar{u}^{2}|G(x, 0)|^{2}\right)\right)\left(1+|G(x, 0)|^{2}\right)}>0, \\
\gamma_{1}=\int_{0}^{2 s} \frac{\bar{\gamma}_{1}(s)}{1+\bar{\gamma}_{1}(1)} d s \\
\bar{\gamma}_{1}(s)=\frac{1}{s} \int_{s}^{2 s}\left(\tilde{\gamma}_{1}(t)-1\right) d t+s, \\
\tilde{\gamma}_{1}(s)=\max _{\left\{(x, u)|x|^{2}+|u|^{2} \leq s\right\}}\left\{1+\int_{0}^{1} \frac{\partial W(x)}{\partial x} \frac{\partial g\left(x, \tau \epsilon_{1} u\right)}{\partial u} d \tau\right\},
\end{gathered}
$$

where $W$ is a $C^{2}$, positive semidefinite function, such that

(1) $L_{f} W \leq 0$;

(2) the system $\dot{x}=f(x)$ is AS conditionally to the set $M=\{x \mid W(x)=0\}$;

(3) for all $\bar{x}$ limiting solutions for the system

$$
\begin{gathered}
\dot{x}=f(x)+g\left(x, \epsilon_{1} u(x)\right)\left(-\lambda(x) \alpha(t) \frac{\partial W}{\partial x}(x) g(x, 0)\right)^{T}, \\
y=h(x)=\lambda(x)^{0.5}\left|\frac{\partial W}{\partial x}(x) g(x, 0)\right|
\end{gathered}
$$

with respect to unbounded sequence $\left\{t_{n}\right\}$ in $[0, \infty)$, then if $h(\bar{x})=0$, a.e., then either $\bar{x}\left(t_{0}\right)=0$ for some $t_{0} \geq 0$ or 0 is a $\omega$-limit point of $\bar{x}$.

Then the closed-loop system (3.26) with (3.27) admits the origin $x=0$ as UAS equilibrium point. Furthermore $|u(x)| \leq \bar{u}, \forall x$.

Proposition 3.14. Consider the closed-loop system that consists of the faulty system (3.4) with the static state feedback:

$$
\begin{aligned}
u(x) & =-\lambda(x) G(x)^{T}, \\
G(x) & =\frac{\partial W(x)}{\partial x} g(x), \\
\lambda(x) & =\frac{2 \bar{u}}{1+|G(x)|^{2}} .
\end{aligned}
$$


where $W$ is a $C^{2}$, positive semidefinite function, such that

(1) $L_{f} W \leq 0$;

(2) the system $\dot{x}=f(x)$ is AS conditionally to the set $M=\{x \mid W(x)=0\}$;

(3) for all $\bar{x}$ limiting solutions for the system

$$
\begin{gathered}
\dot{x}=f(x)+g(x)\left(-\lambda(x) \alpha(t) \frac{\partial W}{\partial x}(x) g(x)\right)^{T}, \\
y=h(x)=\lambda(x)^{0.5}\left|\frac{\partial W}{\partial x}(x) g(x)\right|
\end{gathered}
$$

with respect to unbounded sequence $\left\{t_{n}\right\}$ in $[0, \infty)$, then if $h(\bar{x})=0$, a.e., then either $\bar{x}\left(t_{0}\right)=0$ for some $t_{0} \geq 0$ or 0 is a $\omega$-limit point of $\bar{x}$.

Then the closed-loop system (3.4) with (3.29) admits the origin $x=0$ as UAS equilibrium point. Furthermore $|u(x)| \leq \bar{u}, \forall x$.

These passive NFTC schemes are valid for a large class of nonlinear systems, not necessarily affine in the control, and take into account input saturations; however, the conditions to satisfy might be difficult to check when dealing with models having a large number of states.

\section{Active NFTC}

As we have explained in the introduction, passive FTCs cannot cope with the fault alone, they have to be associated with active FTCs. Indeed, passive FTCs first ensure, at least the stability of the faulty system, during the time period when the FDD is estimating the fault, then active FTC takes over the passive FTC and, using the estimated faulty model they try to optimize the performances of the faulty system. We present in this section some active NFTC schemes.

\subsection{Optimization-Based Active NFTC}

In [14], the authors studied the problem of graceful performance degradation for affine nonlinear systems. The method is an optimization-based scheme, that gives a constructive way to reshape online the output reference for the postfault system, and explicitly take into account the actuators and states saturations. The online output reference reshaping is associated with an online, MPC-based, controller reconfiguration, that forces the postfault system to track the new output reference.

The model considered are affine in the control:

$$
\begin{gathered}
\dot{x}=f(x)+g(x) u, \\
y=h(x),
\end{gathered}
$$

where $x \in \mathbb{R}^{n}, u \in \mathbb{R}^{n_{a}}$, and $y \in \mathbb{R}^{m}$ represent respectively the state, the input and the controlled output vectors. The vector fields $f$, columns of $g$, and function $h$ are supposed to satisfy the following classical assumptions. 
Assumption 4.1. $f: \mathbb{R}^{n} \rightarrow \mathbb{R}^{n}$ and the columns of $g: \mathbb{R}^{n} \rightarrow \mathbb{R}^{n \times n_{a}}$ are smooth vector fields on $a$ compact set $X$ of $\mathbb{R}^{n}$ and $h(x)$ is a smooth function on $X$ with $f(0)=0, h(0)=0$.

Assumption 4.2. System (3.1) has a well-defined (vector) relative degree $\left\{r_{1}, \ldots, r_{m}\right\}$ at each point $x^{0} \in X$ (see e.g., [58]).

Assumption 4.3. The system is fully or over-actuated, in the sense that the number of actuators is at least equal to the number of controlled outputs, that is, $n_{a} \geq m$.

Assumption 4.4. We assume that assumptions 4.1-4.3 above, are preserved after the occurrence of a fault in the system.

Assumption 4.5. We assume additionally that the desired nominal trajectory is feasible by the nominal (safe) system, within its input/state limits.

The control objective is then, that to find a controller $u$ s.t. the nominal as well as the faulty systems' output vector $y$ tracks asymptotically a desired smooth feasible trajectory $y_{d}(t)$, while satisfying the actuators and states constraints:

$$
\begin{gathered}
u \in \Omega \triangleq\left\{u=\left(u_{1}, u_{2}, \ldots, u_{n_{a}}\right)^{T} \mid u_{i}^{-} \leq u_{i} \leq u_{i}^{+}, i=1,2, \ldots, n_{a}\right\} \\
x \in X \triangleq\left\{x=\left(x_{1}, x_{2}, \ldots, x_{n}\right)^{T} \mid x_{i}^{-} \leq x_{i} \leq x_{i}^{+}, i=1,2, \ldots, n\right\}
\end{gathered}
$$

where $u^{-}=\left(u_{1}^{-}, u_{2}^{-}, \ldots, u_{n_{a}}^{-}\right)^{T}, u^{+}=\left(u_{1}^{+}, u_{2}^{+}, \ldots, u_{n_{a}}^{+}\right)^{T}$ and $x^{-}=\left(x_{1}^{-}, x_{2}^{-}, \ldots, x_{n}^{-}\right)^{T}, x^{+}=$ $\left(x_{1}^{+}, x_{2}^{+}, \ldots, x_{n}^{+}\right)^{T}$ are vectors of lower/upper actuators and states limits, respectively. To do so the authors formulate the problem as the following optimization problem:

$$
\min _{\left(a, t_{2 F}\right)} J=\min _{\left(a, t_{2 F}\right)} \int_{t_{1 F}}^{t_{2 F}}\left(y_{\text {nom }}(t)-y_{d}(t)\right)^{T} Q_{1}\left(y_{\text {nom }}(t)-y_{d}(t)\right) d t+\int_{t_{1 F}}^{t_{2 F}} u(t)^{T} Q_{2} u(t) d t
$$

under the constraints

$$
\begin{gathered}
\dot{x}=f_{F}(x)+g_{F}(x) u, \\
y_{d}\left(t, a, t_{2 F}\right)=h(x), \\
u^{-} \leq u \leq u^{+}, \\
x^{-} \leq x \leq x^{+}, \\
y^{(k)}\left(t_{1 F}\right) \triangleq\left(y_{1}^{(k)}\left(t_{1 F}\right), \ldots, y_{m}^{(k)}\left(t_{1 F}\right)\right)^{T}=y_{\text {nom }}^{(k)}\left(t_{1 F}\right) \triangleq\left(y_{\text {nom }_{1}}^{(k)}\left(t_{1 F}\right), \ldots, y_{\text {nom }_{m}}^{(k)}\left(t_{1 F}\right)\right)^{T}, \\
y^{(k)}\left(t_{2 F}\right) \triangleq\left(y_{1}^{(k)}\left(t_{2 F}\right), \ldots, y_{m}^{(k)}\left(t_{2 F}\right)\right)^{T}=y_{\text {nom }}^{(k)}\left(t_{2 \text { nom }}\right) \triangleq\left(y_{\text {nom }_{1}}^{(k)}\left(t_{2 \text { nom }}\right), \ldots, y_{\text {nom }_{m}}^{(k)}\left(t_{2 \mathrm{nom}}\right)\right)^{T}, \\
k=0, \ldots, s, \\
t_{2 F} \geq t_{2 \text { nom }},
\end{gathered}
$$


where $y_{d}(t)=\left(\sum_{i=1}^{i=l+1} a_{i 1}\left(\left(t-t_{1 F}\right) /\left(t_{2 F}-t_{1 F}\right)\right)^{(i-1)}, \ldots, \sum_{i=1}^{i=l+1} a_{\mathrm{im}}\left(\left(t-t_{1 F}\right) /\left(t_{2 F}-t_{1 F}\right)\right)^{(i-1)}\right)^{T} s \in$ $\mathbb{N}^{+}, Q_{1} \in \mathbb{R}^{m \times m}, Q_{2} \in \mathbb{R}^{n_{a} \times n_{a}}$ are positive definite weight matrices, $a=$ $\left(a_{(1) 1}, \ldots, a_{(l+1) 1}, \ldots, a_{(1) m}, \ldots, a_{(l+1) m}\right)^{T} \in \mathbb{R}^{m(l+1)}$ is the vector of the polynomials coefficients, $t_{2 F}$ is the final motion time for the optimal trajectory vector $y_{d}(t)$, and $t_{2 \text { nom }}$ is the final motion time for the nominal trajectory vector $y_{\text {nom }}(t) \triangleq\left(y_{\text {nom }_{1}}(t), \ldots, y_{\text {nom }_{m}}(t)\right)^{T}$, and where $f_{F}, g_{F}$ hold for the modified vector field $f$ and matrix $g$ after the occurrence of the fault. The existence of solutions and the computation scheme was then studied for different cases, that is, without internal dynamics, with internal dynamics for minimum phase and with internal dynamics for nonminimum phase systems. The authors did not consider in this paper explicitly FDD synthesis for nonlinear systems. Instead they assumed the availability of an FDD module and they studied both cases: first where FDD provides a precise postfault model and, second the realistic case, where FDD gives a delayed imprecise postfault model. This optimization-based scheme, can deal with the general class of nonlinear models affine in the control, with state and input constraints, and include a stable inversion part to deal with nonminimum phase systems, however, the necessary online computation can be time consuming for large models.

\subsection{Learning-Based Active NFTC}

We report here the results presented in [59], where the author used a learning scheme to modify the feedback control so as to stabilize the system in the presence of a fault.

The author considers systems of the form

$$
\dot{x}=f(x)+G(x)[u+\eta(x, t)+\beta(t-T) \xi(x)]
$$

where, $x \in \mathbb{R}^{n}$, and $u \in \mathbb{R}^{m}$ are the state and control vectors, respectively, and $G=$ $\left[g_{1}, g_{2}, \ldots, g_{m}\right]$ is an $n \times m$ matrix function, $f, g_{i}: \mathbb{R}^{n} \rightarrow \mathbb{R}^{n} i=1, \ldots, m$ are known smooth vector fields representing the nominal system dynamics, $\beta(t-T)$ is a step function representing an abrupt fault occurring at an unknown time $T, \eta(x, t)$ represents the timevarying model uncertainties, and $\xi(x)$ is the vector of state-dependent faults. The author assumes the existence of a nominal controller $u_{N}(x)$ that guarantees uniform stabilization of the nominal system:

$$
\dot{x}=f(x)+G(x) u .
$$

The scheme assumes also the availability of the closed form Lyapunov function $V_{N}$ associated with the nominal stable feedback system:

$$
\dot{x}=f(x)+G(x) u_{N}(x) .
$$


The author proposes then the NFTC:

$$
\begin{gathered}
u=u_{N}(x)+\phi(x, \widehat{\theta}, \bar{\theta}), \\
\phi(x, \widehat{\theta}, \bar{\theta})=-\Omega(x)^{T} \widehat{\theta}-\bar{\theta} \omega(x), \\
\dot{\hat{\theta}}=\Gamma \Omega(x) p(x), \\
\dot{\bar{\theta}}=\gamma \omega(x)^{T} p(x), \\
p(x)=\left(\frac{\partial V_{N}}{\partial x} G(x)\right)^{T}, \\
\omega_{i}(x)=\tanh \left(\frac{p_{i}(x)}{\epsilon}\right), \quad \epsilon>0, \quad i=1, \ldots, m,
\end{gathered}
$$

where $\Omega(x)$ is a $q \times m$ and represents the basis function for the neural network approximation of the fault $f$ by $\widehat{f}(x, \widehat{\theta})=\Omega(x)^{T} \hat{\theta}, \widehat{\theta} \in \mathbb{R}^{q}$. Then, under the assumption of matching conditions, that is, $\eta, \xi$ are in the range space of $G$, the author proves that the feedback controller (4.8) stabilize the faulty system (4.5). However, this control law, is based on the knowledge of the full state vector, and might lead to chattering effect if the parameter $\epsilon$ is chosen too small.

\subsection{Adaptive Backstepping-Based Active NFTC}

The scheme presented here is based on the results of $[4,50]$. The systems studied are of the form

$$
\begin{gathered}
\dot{x}_{i}=x_{i+1}+\phi_{i}\left(\bar{x}_{i}\right)+\eta_{i}(x, u, t)+\beta_{i}\left(t-T_{0}\right) \xi_{i}\left(\bar{x}_{i}\right), \quad i=1, \ldots, n-1, \\
\dot{x}_{n}=\phi_{0}(x) u+\phi_{n}(x)+\eta_{n}(x, u, t)+\beta_{n}\left(t-T_{0}\right) \xi_{n}(x), \\
y=x_{1}
\end{gathered}
$$

where $x \in \mathbb{R}^{n}$ is the state vector, $\bar{x}_{i}=\left(x_{1}, \ldots, x_{i}\right)^{T}, u \in \mathbb{R}, y \in \mathbb{R}$ are the input and the output, respectively. The function $\phi_{0}$ is a nonzero smooth function, and $\phi_{i}, \eta_{i}, f_{i}, i=1, \ldots, n$ are smooth functions. The control goal is to force the output $y$ to track a desired trajectory $y_{r}(t)$, where $y_{r}^{(l)}, l=0, \ldots, n$ are known, piecewise continuous and bounded. As in Section 4.2, $\eta_{i}, \xi_{i}, i=1, \ldots, n$ represent the model uncertainties and the expected faults, respectively, and $\beta_{i}, i=1, \ldots, n$, represent the time profile of the faults. Then, based on assumption of the availability of a FDD module that detects and estimates the fault, the authors propose the following three-stage controller:

$$
u= \begin{cases}u_{0}\left(x, y_{d}, t\right), & t<T_{d}, \\ u_{D}\left(x, y_{d}, t\right), & T_{d} \leq t<T_{\mathrm{isol}}, \\ u_{I}\left(x, y_{d}, t\right), & T \geq T_{\mathrm{isol}}\end{cases}
$$


where $T_{d}$, and $T_{\text {isol }}$ are the time of the fault detection and fault isolation, respectively. Based on the adaptive-backstepping approach, the authors propose the following expression for the three controllers.

(i) First for $t<T_{d}$

$$
u_{0}(t)=\frac{\alpha_{n}+y_{r}^{(n)}}{\phi_{0}(x)}
$$

with

$$
\begin{aligned}
& \alpha_{0}=0, \\
& \alpha_{1}=-c_{1} z_{1}-c_{2} z_{1}-\phi_{1}, \\
& \alpha_{i}=-c_{1} z_{i}-z_{i-1}-\phi_{i}-c_{2} z_{i} \sum_{j=1}^{i-1}\left(\frac{\partial \alpha_{i-1}}{\partial x_{j}}\right)^{2}+\sum_{j=0}^{i-2} \frac{\partial \alpha_{i-1}}{\partial y_{r}^{(j)}} y_{r}^{(j+1)} \\
& +\sum_{j=1}^{i-1} \frac{\partial \alpha_{i-1}}{\partial x_{j}}\left(x_{j+1}+\phi_{j}\right), \quad i=2, \ldots, n, \\
& z_{i}=x_{i}-\alpha_{i-1}-y_{r}^{(i-1)}, \quad i=1, \ldots, n .
\end{aligned}
$$

(ii) Second for $T_{d} \leq t<T_{\text {isol }}$

$$
u_{D}(t)=\frac{\alpha_{n}+y_{r}^{(n)}}{\phi_{0}(x)}
$$

with

$$
\begin{aligned}
& \alpha_{0}=0 \\
& \begin{aligned}
\alpha_{1}= & -c_{1} z_{1}-\phi_{1}-\widehat{\theta}_{1}^{T} \varphi_{1}+\rho_{1}\left(y, \widehat{\theta}_{1}, \widehat{\psi}, y_{r}\right), \\
\alpha_{i}= & -z_{i-1}-c_{i} z_{i}-\phi_{i}-\widehat{\theta}_{i}^{T} \varphi_{i}\left(\bar{x}_{i}\right)+\sum_{k=1}^{i-1}\left[\frac{\partial \alpha_{i-1}}{\partial x_{k}}\left(x_{k+1}+\phi_{k}+\widehat{\theta}_{k}^{T} \varphi_{k}\left(\bar{x}_{k}\right)\right)\right] \\
& +\sum_{k=1}^{i-1}\left[\frac{\partial \alpha_{i-1}}{\partial y_{r}^{(k-1)}} y_{r}^{(k)}+\frac{\partial \alpha_{i-1}}{\partial \widehat{\theta}_{k}} \tau_{k i}\right]+\sum_{k=1}^{i-1} \frac{\partial \alpha_{i-1}}{\partial x_{k}} \varphi_{k}\left(\bar{x}_{k}\right)^{T} \Gamma_{k} \sum_{l=k}^{i-2}\left(\frac{\partial \alpha_{l}}{\partial \widehat{\theta}_{k}}\right)^{T} z_{l+1}, \\
& +\rho_{i}\left(\bar{x}_{i}, \bar{\theta}_{i}, \widehat{\psi}, \bar{y}_{r}^{(i-1)}\right), \quad i=2, \ldots, n .
\end{aligned}
\end{aligned}
$$

and the parameter adaptive laws are

$$
\begin{gathered}
\dot{\hat{\theta}}_{k}(t)=\tau_{k n}, \quad 1 \leq k \leq n, \\
\dot{\hat{\psi}}=\Gamma_{\psi}\left[\sum_{k=1}^{n} z_{k} \omega_{k}-\sigma\left(\widehat{\psi}-\psi^{0}\right)\right], \quad \psi^{0} \geq 0, \Gamma_{\psi}>0, \sigma>0,
\end{gathered}
$$


with

$$
\begin{gathered}
z_{i}=x_{i}-\alpha_{i-1}-y_{r}^{(i-1)}, \quad i=1, \ldots, n, \\
\tau_{11}=\Gamma_{1}\left[\varphi_{1}\left(x_{1} z_{1}-\sigma\left(\widehat{\theta}_{1}-\theta_{1}^{0}\right)\right)\right], \quad \sigma>0, \Gamma_{1}>0, \\
\tau_{k i}=\tau_{k(i-1)}-\Gamma_{k} z_{i} \frac{\partial \alpha_{i-1}}{\partial x_{k}} \varphi_{k}\left(\bar{x}_{k}\right), \quad \Gamma_{k}>0,1 \leq k \leq i-1, i=2, \ldots, n, \\
\tau_{i i}=\Gamma_{i}\left[\varphi_{i}\left(\bar{x}_{k}\right) z_{i}-\sigma\left(\widehat{\theta}_{i}-\theta_{i}^{0}\right)\right], \quad \Gamma_{i}>0, i=2, \ldots, n .
\end{gathered}
$$

where $\varphi_{i}, i=1, \ldots, n$ are the basis functions of the linear approximation for the unknown fault function, that is, $\widehat{\xi}_{i}\left(\bar{x}_{i}, \widehat{\theta}_{i}\right)=\left(\widehat{\theta}_{i}\right)^{T} \varphi_{i}\left(\bar{x}_{i}\right)$, and $\rho_{i}, i=1, \ldots, n$ are given bounding control functions.

(iii) Third for $t \geq T_{\text {isol }}$

$$
\begin{gathered}
u_{I}(t)=\frac{\alpha_{n}+y_{r}^{(n)}}{\phi_{0}(x)}, \\
\dot{\hat{\theta}_{k}}(t)=\tau_{k n^{\prime}}^{I} \quad 1 \leq k \leq n, \\
\dot{\hat{\psi}}=\Gamma_{\psi}\left[\sum_{k=1}^{n} z_{k} \omega_{k}-\sigma\left(\widehat{\psi}-\psi^{0}\right)\right], \quad \psi^{0} \geq 0, \Gamma_{\psi}>0, \sigma>0,
\end{gathered}
$$

associated with the same update laws (4.16), except that the basis functions and the bounding control functions are different from the previous case, that is, for $u_{D}$, since in this case they are specific to the isolated fault. Then, the authors proved that under the assumption of bounded uncertainties $\eta_{i}(x, u, t)$ and bounded fault approximation-error, that is, $\xi_{i}\left(\bar{x}_{i}\right)-\widehat{\xi}_{i}\left(\bar{x}_{i}, \widehat{\theta}_{i}\right)$ bounded $\forall i$, that all the signals and parameter estimates are uniformly bounded, that is, $z(t), \hat{\theta}(t), \hat{\psi}(t)$, and $x(t)$ are bounded $\forall t$. However, this approach is based on the special structure of the faulty model (4.9), and assumes the availability of the measurements of state vector for the feedback control. Eventually, the FDD and FTC presented here are based on the assumption of the fault being part of an apriori known set of expected fault's models.

\subsection{Switched Control-Based Active NFTC}

We report here the schemes introduced in $[25,26]$, where the authors consider both problems of FDD and FTC for a class of nonlinear systems, with input constraints. The model studied are of the form

$$
\begin{gathered}
\dot{x}=f(x)+G_{k(t)}(x)\left(u_{k(t)}(y)+\tilde{u}_{k(t)(t)}\right), \\
y(x)=h(x), \quad u_{k}(t) \in U_{k}, \quad u_{k(t)}(y)+\tilde{u}_{k(t)(t)} \in U_{k}, \\
k(\mathrm{t}) \in K=\{1, \ldots N\}, \quad N<\infty, \quad U_{k}=\left\{u \in \mathbb{R}^{m}:|u| \leq u_{k}^{\max }\right\}, \quad u_{k}^{\max }>0 \quad \forall k,
\end{gathered}
$$

where $x \in \mathbb{R}^{n}$ is the vector of state variables, $y \in \mathbb{R}^{m}$ is the vector of measurable variables, and $u_{k}(y) \in \mathbb{R}^{m}$ denotes the control vector under the $k$ th configuration. The additive actuator faults are modelled by $\tilde{u}_{k}$. The vector function $f$ and the matrices $G_{k}(x), \forall k$ are assumed 
to be sufficiently smooth on their domains of definition. For each value of $k \in K$ the system is controlled via a different set of manipulated inputs, which defines a given control configuration. The nonlinear model (4.18) is associated with the following assumption.

Assumption 4.6. Consider the system (4.18) in configuration $k$ under state-feedback. Then for every input $u_{j, k}, j=1, \ldots, m$, there exists a unique state $x_{i, k}, i=1, \ldots, n$, such that with $x_{i, k}$ as output, the relative degree of $x_{i, k}$ with respect to $u_{j, k}$ and only with respect to $u_{j . k}$ is equal to 1 .

This assumption means that each actuator is the only one influencing at least some state. This implies that the effect of a specific actuator on the system evolution is completely distinguishable, which allows fault isolation in that specific actuator. This sufficient fault detection/isolation condition, can be relaxed if the input enters the model in an "uppertriangular" or "lower-triangular" form (refer to [25, Remark 3]). The authors introduced a nonlinear FDD in the following theorem.

Theorem 4.7. Consider the model (4.18) in configuration $k$ which satisfies Assumption 4.6, under the control law:

$$
\begin{gathered}
u_{k}=-\omega_{k}\left(x, u_{k}^{\max }\right)\left(L_{G_{k}} V_{k}(x)\right)^{T}, \\
\omega_{k}\left(x, u_{k}^{\max }\right)= \begin{cases}\frac{\alpha_{k}(x)+\sqrt{\alpha_{k}^{2}(x)+\left(u_{k}^{\max }\left|b_{k}^{T}(x)\right|\right)^{4}}}{\left|b_{k}^{T}(x)\right|^{2}\left(1+\sqrt{1+\left(u_{k}^{\max }\left|b_{k}^{T}(x)\right|^{2}\right)^{2}}\right)^{2}} & b_{k}^{T}(x) \neq 0, \\
0, & b_{k}^{T}(x)=0,\end{cases} \\
\alpha_{k}(x)=L_{f_{k}} V_{k}(x)+\rho_{k} V_{k}(x), \quad \rho_{k}>0, \\
b_{k}(x)=L_{G_{k}} V_{k}(x) .
\end{gathered}
$$

assuming that the set $\Phi_{k}\left(u_{k}^{\max }\right)=\left\{x\right.$, s.t. $\left.L_{f_{k}} V_{k}(x)+\rho_{k} V_{k}(x) \leq u_{k}^{\max }\left|\left(L_{G_{k}} V_{k}(x)\right)^{T}\right|\right\}$, contains the origin and a neighborhood of the origin.

Let the fault detection and isolation filter for the $j$ th manipulated input in the $k$ th configuration be described by

$$
\begin{gathered}
\dot{\tilde{x}}_{i, k}=f_{i}\left(x_{1}, \ldots, \tilde{x}_{i, k}, \ldots, x_{n}\right)+g_{j, k}[i]\left(x_{1}, \ldots, \tilde{x}_{i, k}, \ldots, x_{n}\right) \times u_{j, k}\left(x_{1}, \ldots, \tilde{x}_{i, k}, \ldots, x_{n}\right) \\
e_{i, k}=\tilde{x}_{i, k}-x_{i},
\end{gathered}
$$

where $g_{j, k}[i]$ denotes the $i$ th element of the vector $g_{j, k}, \tilde{x}_{i, k}(0)=x_{i}(0)$ and the subscripts $i, k$ refer to the $i$ th state under the $k$ th control configuration. Let $T_{j, k}^{f}$ be the earliest time for which $\tilde{u}_{j, k} \neq 0$, then the fault detection and isolation filter of (4.20) ensures that $\lim _{t \rightarrow T_{j, k}^{f+}} e_{i, k}(t) \neq 0$. Also, $e_{i, k}(t) \neq 0$ only if $\tilde{u}_{j, k}(s) \neq 0,0 \leq s<t$.

Then, the NFTC has been introduced in the following theorem. 
Theorem 4.8. Consider the closed-loop system (4.18), (4.19), and let $x(0) \in \Omega_{k_{0}}$ for some $k_{0} \in K$, with $\Omega_{k}$ being defined as: $\Omega_{k}\left(u_{k}^{\max }\right)=\left\{x \in \mathbb{R}^{n}: V_{k}(x) \leq c_{k}^{\max }\right\} \subset \Phi_{k}, c_{k}^{\max }>0$ is a level set of $V_{k}$. Let $T_{j, k_{0}}$ be the earliest time such that $e_{i, k_{0}} \neq 0$ for some $i$ corresponding to a manipulated input $u_{j, k_{0}}$ in (4.20). Then, the following switching rule:

$$
k(t)=\left\{\begin{array}{l}
k_{0}, \quad 0 \leq t<T_{j, k_{0},} \\
q \neq k_{0}, \quad t \geq T_{j, k_{0}}, \quad x\left(T_{j, k_{0}}\right) \in \Omega_{q}, \quad u_{j, k_{0}} \notin u_{q},
\end{array}\right.
$$

guarantees asymptotic stability of the origin of the closed-loop system (4.18) and (4.19).

This active NFTC are applicable for the general class of nonlinear models affine in the control, and are based on a state-feedback (the authors proposed in the same papers an extension to the case of output feedback). However, they require Assumption 4.6 to hold to be able to detect and isolate the actuator fault. Another point is that this scheme does not consider multiplicative actuator faults.

\subsection{Predictive Control-Based Active NFTC}

The authors in $[28,29]$ study the problem of NFTC for nonlinear models affine in the control, with input constraints and uncertainties. The nature of faults treated is actuator faults, under the assumption of controllability of the faulty system. Let us recall below the main result of these work.

The models considered are of the form

$$
\begin{gathered}
\dot{x}=f(x)+G_{k}(x) u_{k}+W_{k}(x) \theta_{k}(t), \quad u_{k} \in U_{k}, \theta_{k} \in \Theta_{k}, \\
k \in\{1, \ldots, N\}, \quad N<\infty
\end{gathered}
$$

where $x \in \mathbb{R}^{n}$ denotes the vector of state variables, $u \in U_{k} \subset \mathbb{R}^{m}, U_{k}=\left\{u \in \mathbb{R}^{m}\right.$, s.t. $|u| \leq$ $\left.u_{k}^{\max }\right\}$, and $u_{k}^{\max }>0 \forall k$ denotes the vector of constrained inputs. The vector $\theta_{k}(t)=$ $\left[\theta_{k}^{1} \cdots \theta_{k}^{q}\right]^{T} \in \Theta_{k} \subset \mathbb{R}^{q}$ denotes the vector of time-varying uncertainties but bounded variables taking values in a nonempty compact convex subset of $\mathbb{R}^{q}$. The vector $f(x)$ (s.t. $f(0)=0$ ), the matrices $G_{k}(x)=\left[g_{k}^{1}(x) \cdots g_{k}^{m}(x)\right], g_{k}^{i} \in \mathbb{R}^{n}, i=1 \cdots m$, and $W(x)=\left[w_{k}^{1}(x) \cdots w_{k}^{q}(x)\right], w_{k}^{i} \in$ $\mathbb{R}^{n}, i=1 \cdots q$, are assumed to be sufficiently smooth on their domain of definition. For each value of the index $k$ the process is controlled via a different manipulated input, which defines a given control configuration. Switching between the available $N$ control configuration is controlled by a higher-level supervisor, which ensures that only one control configuration is active at any given time, and allows only finite number of switches over any finite time interval of time. The main idea of this work is that the authors assume that after the occurrence of a fault, the system will be associated with one of the $N$ configuration and then they build off-line a bank of $N$ nonlinear model-predictive stabilizing controllers, and based on the value of the state vector at the time of fault occurrence, they switch among these controllers to ensure the stability of the faulty system. To make the presentation of the NFTC clear, we follow the same structure of the paper [28] and present first a Lyapunov-based switched controller, then we present the associated nonlinear model predictive controller (NMPC) and finally we present the NFTC based on this algorithm. 
The Lyapunov-based controller associated with the system (4.22) is given by the bounded state feedback:

$$
u_{k}^{b}=-\frac{\alpha_{k}(x)+\sqrt{\alpha_{1, k}(x)^{2}+\left(u_{k}^{\max } \beta_{k}(x)\right)^{4}}}{\beta_{k}(x)^{2}\left(1+\sqrt{1+\left(u_{k}^{\max } \beta_{k}(x)\right)^{2}}\right)}\left(L_{G_{k} V_{k}}\right)^{T},
$$

where, $V_{k}$ is a robust control Lyapunov function (RCLF) (as defined in [60, page 49]), $\alpha_{k}(x)=$ $L_{f} V_{k}+\left(\rho_{k}\|x\|+\chi_{k} \theta_{k}^{b}\left\|L_{W_{k}} V_{k}\right\|\right)\left(\|x\| /\left(\|x\|+\phi_{k}\right)\right), \alpha_{1, k}(x)=L_{f} V_{k}+\rho_{k}\|x\|+\chi_{k} \theta_{k}^{b}\left\|L_{W_{k}} V_{k}\right\|, \beta_{k}(x)=$ $\left\|L_{G_{k}} V_{k}\right\|, L_{G_{k}} V_{k}=\left[L_{g_{k}^{1}} V_{k} \cdots L_{g_{k}^{m}} V_{k}\right], L_{W_{k}} V_{k}=\left[L_{W_{k}^{1}} V_{k} \cdots L_{W_{k}^{q}} V_{k}\right], \theta_{k}^{b}>0$, s.t. $\left\|\theta_{k}(t)\right\| \leq \theta_{k^{\prime}}^{b}, \forall t$, and $\rho_{k}>0, x_{k}>1, \phi_{k}>0$.

The following convergence result has been reported in [28] and proven in [61]: let $\Pi_{k}\left(\theta_{k}^{b}, u_{k}^{\max }\right)=\left\{x \in \mathbb{R}^{n}: \alpha_{1, k}(x) \leq u_{k}^{\max } \beta_{k}(x)\right\}$ and assume that $\Omega_{k}=\left\{x \in \mathbb{R}^{n}: V_{k}(x) \leq\right.$ $\left.c_{k}^{\max }\right\} \subseteq \Pi_{k}\left(\theta_{k}^{b}, u_{k}^{\max }\right)$, for some $c_{k}^{\max }>0$. Then, given any positive real number, $d_{k}^{r}$, s.t.: $\mathbb{D}_{k}^{r}=$ $\left\{x \in \mathbb{R}^{n}:\|x\| \leq d_{k}^{r}\right\} \subset \Omega_{k}$ and $\forall x_{0} \in \Omega_{k}, \exists \epsilon_{k}^{r *}>0$, s.t. if $\phi_{k} /\left(\chi_{k}-1\right)<\epsilon_{k}^{r *}$ the solutions of the closed-loop system (4.22) and (4.23) satisfy $x(t) \in \Omega_{k}, \forall t$ and $\lim \sup _{t \rightarrow \infty}\|x(t)\| \leq d_{k}^{r}$.

We also need to recall a convergence result from [28], that characterizes the behavior of the solutions of (4.22) and (4.23), when the continuous controller (4.23) is implemented in discrete time. The result is as follows: consider the system (4.22) for a fixed $k$ with $\theta_{k}=0, \forall t$, associated with the controller (4.23). Let $u_{k}(t)=u_{k}^{b}\left(j \Delta_{k}\right), j \Delta_{k} \leq t<(j+1) \Delta_{k}, j=0, \ldots, \infty$. Then, $\forall d_{k}>0, \exists \Delta_{k}^{*}>0, \delta_{k}^{\prime}>0, \epsilon_{k}^{*}>0$ s.t. if $\Delta_{k} \in\left(0, \Delta_{k}^{*}\right]$ and $x(0) \in \Omega_{k}$ then $x(t) \in \Omega_{k} \forall t$ and $\limsup \sup _{t \rightarrow \infty}\|x(t)\| \leq d_{k}$. Also, if $V_{k}(x(0)) \leq \delta_{k}^{\prime}$ then $V_{k} \leq \delta_{k}^{\prime} \forall \tau \in\left[0, \Delta_{k}\right)$ and if $\delta_{k}^{\prime}<$ $V_{k}(x(0)) \leq c_{k}^{\max }$, then $\dot{V}_{k}(x(\tau)) \leq-\epsilon_{k}^{*} \quad \forall \tau\left[0, \Delta_{k}\right)$.

Next we report the Lyapunov-based predictive control associated with the Lyapunovbased controller (4.23). The following result has been reported in [28] and proven in [62]:

Consider the system (4.22), for a fixed value of $k$, with $\theta_{k}(t)=0, \forall t$, associated with the following NMPC controller:

$$
\begin{aligned}
& \min \left\{J\left(x, t, u_{k}\right), u_{k} \in S_{k}\right\}, \\
& J\left(x, t, u_{k}\right)=\int_{t}^{t+T}\left(\left\|x^{u}(s, x, t)\right\|_{Q_{k}}^{2}+\left\|u_{k}\right\|_{R_{k}}^{2}\right) d s, \quad Q_{k} \geq 0, R_{k}>0, \\
& \text { s.t. } \quad \dot{x}=f_{k}(x)+G_{k}(x) u_{k}, \\
& \dot{V}_{k}(x(\tau)) \leq-\epsilon_{k} \quad \text { if } V_{k}(x(t))>\delta_{k^{\prime}}^{\prime} \quad \tau[t, t+\Delta k), \\
& V_{k}(x(\tau)) \leq \delta_{k}^{\prime} \quad \text { if } V_{k}(x(t)) \leq \delta_{k^{\prime}}^{\prime} \quad \tau \in[t, t+\Delta k),
\end{aligned}
$$

where $\epsilon_{k}, \delta_{k}^{\prime}$ are as defined above, $S_{k}$ is the family of piecewise continuous functions with period $\Delta_{k}$ mapping $[t, t+T]$ into $U_{k}, T>0$ is the horizon of the optimization, and $V_{k}$ is RCLF that yields a stability region $\Omega_{k}$, under continuous implementation of the controller (4.23), with a fixed $\rho_{k}>0$. Then, $\forall d_{k}>0, \exists \Delta_{k}^{*}>0$, and $\delta_{k}^{\prime}>0$, s.t., if $x(0) \in \Omega_{k}$ and $\Delta \in\left(0, \Delta_{k}^{*}\right]$, then $x(t) \in \Omega_{k}, \forall t$ and limsup $\sup _{t \rightarrow \infty}\|x(t)\| \leq d_{k}$.

Finally, we can report the predictive control-based NFTC as follows: Consider the system (4.22), for which the bounded controllers (4.23) and Lyapunov-based MPCs (4.24) have been designed and the stability regions $\Omega_{j}, j=1, \ldots, \mathrm{N}$, under the Lyapunov-based MPCs have been explicitly characterized. Let $d_{\max }=\max _{j=1, \ldots, N} d_{j}, d_{j}$ as defined above, and 
let $\Omega_{U}=\cup_{j=1}^{j=N} \Omega_{j}$. Define $J_{j}(t)=\int_{t}^{t+T_{j}}\left(\left\|x^{u}(s, x, t)\right\|_{Q_{k}}^{2}+\left\|u_{k}^{b}\right\|_{R_{k}}^{2}\right) d s$, where $t+T_{j} \geq t$ is the earliest time at which the state of the closed-loop system under bounded controller enters the level set defined by $V_{j}(x)=\delta_{j}^{\prime}$. Then, let $k(0)=i$ for some index $i \in\{1, \ldots, N\}$ and $x(0) \in \Omega_{i}$. Let $T_{i}^{f}$ be the earliest time at which a fault occurs. Furthermore, let $f=\left\{j:\right.$ s.t. $\left.j \neq i, x\left(T_{i}^{f}\right) \in \Omega_{j}\right\}$, and let $l$ be such that $J_{l}=\min _{j \in f} J_{j}$. Then, the following switching rule

$$
k(t) \begin{cases}i, & 0 \leq t<T_{i}^{f}, \\ l, & t \geq T_{i}^{f}\end{cases}
$$

guarantees that $x(t) \in \Omega_{U}, \forall t \geq 0$ and $\limsup _{t \rightarrow \infty}\|x(t)\| \leq d_{\max }$.

To avoid further overload this paper with long equations, we have reported here only the NFTC in the case without uncertainties, that is, $\theta_{k}(t)=0, \forall k, \forall t$. The interested reader may refer to the references $[28,29]$ for the uncertain case.

This active NFTC, based on the computation off-line of a bank of robust nonlinear controllers, is valid for general nonlinear models affine in the control, however, it is based on the availability of a robust control Lyapunov function in closed-from, which is usually not easily accessible [60]. We can also point out, that in these work [28, 29], the authors assumed the availability of a FDD bloc, and did not consider the problems of fault isolation and estimation delays as well as FDD uncertainties.

\section{Conclusion}

In the last decades there have been a myriad of results on FTC. Many of those work concentrated initially on linear FTC, and more and more researches started focussing on the nonlinear FTC problems, the later being more challenging than the linear FTC because of the difficulties intrinsic to nonlinear systems. However, many encouraging results have been obtained. We wanted to summarize in this paper the results obtained recently on NFTC. We recalled in the introduction most of the FTC work on nonlinear models. We reported the detailed controllers of some of these results. Unfortunately, it was not possible to report in details all the available results. Our choice was mainly motivated by the degree of the "model-nonlinearities", and we reported the work that, in our opinion, treated some general degree of nonlinearities. Although many interesting results have been obtained so far, we believe that work treating together both problems of nonlinear FDD and nonlinear FTC in an effective applicable methods, are still missing. Real-life applications of those NFTC theories are also a missing part of the recent work. To conclude, the case of infinite dimension nonlinear models, that is, nonlinear partial derivative equations-based models, has yet to be studied, some recent results in this directions are presented in [63-66].

\section{References}

[1] Y. Zhang and J. Jiang, "Issues on integration of fault diagnosis and reconfigurable control in active fault-tolerant control systems," in Proceedings of the 6th IFAC Symposium of Fault Detection Supervision and Safety for Technical Processes, pp. 1513-1524, Beijing, China, August-September 2006.

[2] M. Staroswiecki, H. Yang, and B. Jiang, "Progressive accommodation of aircraft actuator faults," in Proceedings of the 6th IFAC Symposium of Fault Detection Supervision and Safety for Technical Processes, pp. 877-882, Beijing, China, August-September 2006. 
[3] H. Niemann and J. Stoustrup, "Passive fault tolerant control of a double inverted pendulum-a case study," Control Engineering Practice, vol. 13, no. 8, pp. 1047-1059, 2005.

[4] X. Zhang, T. Parisini, and M. M. Polycarpou, "Adaptive fault-tolerant control of nonlinear uncertain systems: an information-based diagnostic approach," IEEE Transactions on Automatic Control, vol. 49, no. 8, pp. 1259-1274, 2004.

[5] A. Ingimundarson and R. Sanchez-Pena, "Using the unfalsified control concept to achieve fault tolerance," in Proceedings of the 17th IFAC Word Congress, pp. 1236-1242, Seoul, Korea, July 2008.

[6] F. Liao, J. L. Wang, and G.-H. Yang, "Reliable robust flight tracking control: an LMI approach," IEEE Transactions on Control Systems Technology, vol. 10, no. 1, pp. 76-89, 2002.

[7] S. Wu, M. Grimble, and W. Wei, "QFT based robust/fault tolerantflight control design for a remote pilotless vehicle," in Proceedings of the IEEE International Conference on Control Applications, vol. 1, pp. 57-62, Kohala Coast, Hawaii, USA, August 1999.

[8] S.-F. Wu, M. J. Grimble, and W. Wei, "QFT-based robust/fault-tolerant flight control design for a remote pilotless vehicle," IEEE Transactions on Control Systems Technology, vol. 8, no. 6, pp. 1010-1016, 2000.

[9] H. Niemann and J. Stoustrup, "Reliable control using the primary and dual Youla parametrization," in Proceedings of the 41st IEEE Conference on Decision and Control, vol. 4, pp. 4353-4358, 2002.

[10] M. Benosman and K.-Y. Lum, "Application of absolute stability theory to robust control against loss of actuator effectiveness," IET Control Theory \& Applications, vol. 3, no. 6, pp. 772-788, 2009.

[11] C. Bonivento, A. Isidori, L. Marconi, and A. Paoli, "Implicit fault-tolerant control: application to induction motors," Automatica, vol. 40, no. 3, pp. 355-371, 2004.

[12] C. Bonivento, L. Gentili, and A. Paoli, "Internal model based fault tolerant control of a robot manipulator," in Proceedings of the 43rd IEEE Conference on Decision and Control, vol. 5, pp. 5260-5265, 2004.

[13] M. Benosman and K.-Y. Lum, "Passive actuators' fault-tolerant control for affine nonlinear systems," in Proceedings of the 17th IFAC Word Congress, pp. 14229-14234, Seoul, Korea, July 2008.

[14] M. Benosman and K.-Y. Lum, "Online references reshaping and control reallocation for nonlinear fault tolerant control," IEEE Transactions on Control Systems Technology, vol. 17, no. 2, pp. 366-379, 2009.

[15] M. Benosman and K.-Y. Lum, "Application of passivity and cascade structure to robust control against loss of actuator effectiveness," to appear in International Journal of Robust and Nonlinear Control.

[16] Y. Zhang and J. Jiang, "Design of integrated fault detection, diagnosis and reconfigurable control systems," in Proceedings of the 38th IEEE Conference on Decision and Control, pp. 3587-3592, Phoenix, Ariz, USA, 1999.

[17] Y. Zhang and J. Jiang, "Integrated design of reconfigurable fault-tolerant control systems," Journal of Guidance, Control, and Dynamics, vol. 24, no. 1, pp. 133-136, 2001.

[18] G. Tao, S. Chen, and S. M. Joshi, "An adaptive actuator failure compensation controller using output feedback," IEEE Transactions on Automatic Control, vol. 47, no. 3, pp. 506-511, 2002.

[19] N. E. Wu, Y. Zhang, and K. Zhou, "Detection, estimation, and accommodation of loss of control effectiveness," International Journal of Adaptive Control and Signal Processing, vol. 14, no. 7, pp. 775795, 2000.

[20] B. Jiang and F. N. Chowdhury, "Fault estimation and accommodation for linear MIMO discrete-time systems," IEEE Transactions on Control Systems Technology, vol. 13, no. 3, pp. 493-499, 2005.

[21] M. Mahmoud, J. Jiang, and Y. Zhang, "Stabilization of active fault tolerant control systems with imperfect fault detection and diagnosis," Stochastic Analysis and Applications, vol. 21, no. 3, pp. 673701, 2003.

[22] M. V. Basin and M. A. Pinsky, "Stability impulse control of faulted nonlinear systems," IEEE Transactions on Automatic Control, vol. 43, no. 11, pp. 1604-1608, 1998.

[23] B. Jiang and F. N. Chowdhury, "Parameter fault detection and estimation of a class of nonlinear systems using observers," Journal of the Franklin Institute, vol. 342, no. 7, pp. 725-736, 2005.

[24] B. Jiang, M. Staroswiecki, and V. Cocquempot, "Fault accommodation for nonlinear dynamic systems," IEEE Transactions on Automatic Control, vol. 51, no. 9, pp. 1578-1583, 2006.

[25] P. Mhaskar, C. McFall, A. Gani, P. D. Christofides, and J. F. Davis, "Isolation and handling of actuator faults in nonlinear systems," Automatica, vol. 44, no. 1, pp. 53-62, 2008.

[26] P. Mhaskar, A. Gani, N. H. El-Farra, C. McFall, P. D. Christofides, and J. F. Davis, "Integrated faultdetection and fault-tolerant control of process systems," AIChE Journal, vol. 52, no. 6, pp. 2129-2148, 2006.

[27] A. Fekih, "Effective fault tolerant control design for nonlinear systems: application to a class of motor control system," IET Control Theory \& Applications, vol. 2, no. 9, pp. 762-772, 2008. 
[28] P. Mhaskar, A. Gani, and P. D. Christofides, "Fault-tolerant control of nonlinear processes: performance-based reconfiguration and robustness," International Journal of Robust and Nonlinear Control, vol. 16, no. 3, pp. 91-111, 2006.

[29] P. Mhaskar, "Robust model predictive control design for fault-tolerant control of process systems," Industrial and Engineering Chemistry Research, vol. 45, no. 25, pp. 8565-8574, 2006.

[30] H.-J. Ma and G.-H. Yang, "FTC synthesis for nonlinear systems: sum of squares optimization approach," in Proceedings of the IEEE Conference on Decision and Control, pp. 2645-2650, New Orleans, La, USA, 2007.

[31] M. A. Demetriou, K. Ito, and R. C. Smith, "Adaptive monitoring and accommodation of nonlinear actuator faults in positive real infinite dimensional systems," IEEE Transactions on Automatic Control, vol. 52, no. 12, pp. 2332-2338, 2007.

[32] P. G. de Lima and G. G. Yen, "Accommodating controller malfunctions through fault tolerant control architecture," IEEE Transactions on Aerospace and Electronic Systems, vol. 43, no. 2, pp. 706-722, 2007.

[33] M. Benosman and K. Lum, “On-line references reshaping and control re-allocation for nonminimum phase nonlinear fault tolerant control," in Proceedings of the 17th IFAC Word Congress, pp. 2563-2569, Seoul, Korea, July 2008.

[34] A. Shumsky, "Algebraic approach to the problem of fault accommodation in nonlinear systems," in Proceedings of the 17th IFAC Word Congress, pp. 1884-1889, Seoul, Korea, July 2008.

[35] H. Yang, V. Cocquempot, and B. Jiang, "Robust fault tolerant tracking control with application to hybrid nonlinear systems," IET Control Theory E Applications, vol. 3, no. 2, pp. 211-224, 2009.

[36] Y. Zhang and J. Jiang, "Bibliographical review on reconfigurable fault-tolerant control systems," in Proceeding of the 5th IFAC Symposium on Fault Detection, Supervision and Safety for Technical Processes, pp. 265-276, Washington, DC, USA, 2003.

[37] R. Patton, "Fault-tolerant control systems: the 1997 situation," in IFAC Symposium on Fault Detection Supervision and Safety for Technical Processes, pp. 1033-1055, Hull, UK, 1997.

[38] J. Lunze and J. H. Richter, "Reconfigurable fault-tolerant control: a tutorial introduction," European Journal of Control, vol. 14, no. 5, pp. 359-386, 2008.

[39] M. Benosman and K.-Y. Lum, "Passive actuators' fault tolerant control for affine nonlinear systems," to appear in IEEE Transactions on Control Systems Technology.

[40] Q. Zhao and J. Jiang, "Reliable state feedback control system design against actuator failures," Automatica, vol. 34, no. 10, pp. 1267-1272, 1998.

[41] P. S. Maybeck, "Multiple model adaptive algorithms for detecting and compensating sensor and actuator/surface failures in aircraft flight control systems," International Journal of Robust and Nonlinear Control, vol. 9, no. 14, pp. 1051-1070, 1999.

[42] V. Dardinier-Maron, F. Hamelin, and H. Noura, "A fault-tolerant control design against major actuator failures: application to a three-tank system," in Proceedings of the 38th IEEE Conference on Decision and Control, vol. 4, pp. 3569-3574, Phoenix, Ariz, USA, 1999.

[43] G. Tao, S. M. Joshi, and X. Ma, "Adaptive state feedback and tracking control of systems with actuator failures," IEEE Transactions on Automatic Control, vol. 46, no. 1, pp. 78-95, 2001.

[44] M. Maki, J. Jiang, and K. Hagino, "A stability guaranteed active fault-tolerant control system against actuator failures," International Journal of Robust and Nonlinear Control, vol. 14, no. 12, pp. 1061-1077, 2004.

[45] J. Lunze and T. Steffen, “Control reconfiguration after actuator failures using disturbance decoupling methods," IEEE Transactions on Automatic Control, vol. 51, no. 10, pp. 1590-1601, 2006.

[46] W. Chen and M. Saif, "Adaptive actuator fault detection, isolation and accommodation in uncertain systems," International Journal of Control, vol. 80, no. 1, pp. 45-63, 2007.

[47] F. Bateman, H. Noura, and M. Ouladsine, "A fault tolerant control strategy for an unmanned aerial vehicle based on a sequential quadratic programming algorithm," in Proceedings of the 47th IEEE Conference on Decision and Control, pp. 423-428, Cancun, Mexico, 2008.

[48] T. Miksch, A. Gambier, and E. Badreddin, "Real-time implementation of fault-tolerant control using model predictive control," in Proceedings of the 17th IFAC Word Congress, pp. 11136-11141, Seoul, Korea, July 2008.

[49] J. Richter and J. Lunze, "Reconfigurable control of Hammerstein systems after actuator faults," in Proceedings of the 17th IFAC Word Congress, pp. 3210-3215, Seoul, Korea, July 2008.

[50] X. Zhang, M. M. Polycarpou, and T. Parisini, "Integrated design of fault diagnosis and accommodation schemes for a class of nonlinear systems," in Proceedings of the 40th IEEE Conference on Decision and Control, vol. 2, pp. 1448-1453, Orlando, Fla, USA, 2001. 
[51] D. Ye and G. H. Yang, "Adaptive fault-tolerant control for a class of nonlinear systems with time delay," International Journal of Systems Science, vol. 39, no. 1, pp. 43-56, 2008.

[52] R. Sepulchre, M. Janković, and P. V. Kokotović, Constructive Nonlinear Control, Communications and Control Engineering Series, Springer, Berlin, Germany, 1997.

[53] C. I. Byrnes, A. Isidori, and J. C. Willems, "Passivity, feedback equivalence, and the global stabilization of minimum phase nonlinear systems," IEEE Transactions on Automatic Control, vol. 36, no. 11, pp. 1228-1240, 1991.

[54] T.-C. Lee and Z.-P. Jiang, "A generalization of Krasovskii-LaSalle theorem for nonlinear time-varying systems: converse results and applications," IEEE Transactions on Automatic Control, vol. 50, no. 8, pp. 1147-1163, 2005.

[55] H. Khalil, Nonlinear Systems, Prentice-Hall, Upper Saddle River, NJ, USA, 3rd edition, 2002.

[56] A. Teel and L. Praly, "Tools for semiglobal stabilization by partial state and output feedback," SIAM Journal on Control and Optimization, vol. 33, no. 5, pp. 1443-1488, 1995.

[57] M. Vidyasagar, Nonlinear Systems Analysis, Prentice-Hall, Upper Saddle River, NJ, USA, 2nd edition, 1993.

[58] A. Isidori, Nonlinear Control Systems, Communications and Control Engineering Series, Springer, Berlin, Germany, 2nd edition, 1989.

[59] M. M. Polycarpou, "Fault accommodation of a class of multivariable nonlinear dynamical systems using a learning approach," IEEE Transactions on Automatic Control, vol. 46, no. 5, pp. 736-742, 2001.

[60] R. A. Freeman and P. V. Kokotović, Robust Nonlinear Control Design: State-Space and Lyapunov Technique, Systems \& Control: Foundations \& Applications, Birkhäuser, Boston, Mass, USA, 1996.

[61] N. H. El-Farra and P. D. Christofides, "Coordinating feedback and switching for control of hybrid nonlinear processes," AIChE Journal, vol. 49, no. 8, pp. 2079-2098, 2003.

[62] P. Mhaskar, N. H. El-Farra, and P. D. Christofides, "Predictive control of switched nonlinear systems with scheduled mode transitions," IEEE Transactions on Automatic Control, vol. 50, no. 11, pp. 16701680, 2005.

[63] A. Armaou and M. A. Demetriou, "Robust detection and accommodation of incipient component and actuator faults in nonlinear distributed processes," AIChE Journal, vol. 54, no. 10, pp. 2651-2662, 2008.

[64] N. H. El-Farra, M. A. Demetriou, and P. D. Christofides, "Actuator and controller scheduling in nonlinear transport-reaction processes," Chemical Engineering Science, vol. 63, no. 13, pp. 3537-3550, 2008.

[65] N. H. El-Farra, "Integrated fault detection and fault-tolerant control architectures for distributed processes," Industrial and Engineering Chemistry Research, vol. 45, no. 25, pp. 8338-8351, 2006.

[66] N. H. El-Farra and S. Ghantasala, "Actuator fault isolation and reconfiguration in transport-reaction processes," AIChE Journal, vol. 53, no. 6, pp. 1518-1537, 2007. 


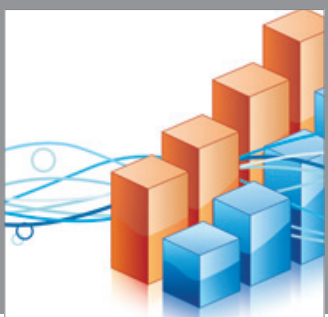

Advances in

Operations Research

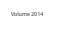

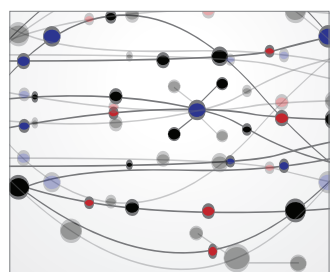

\section{The Scientific} World Journal
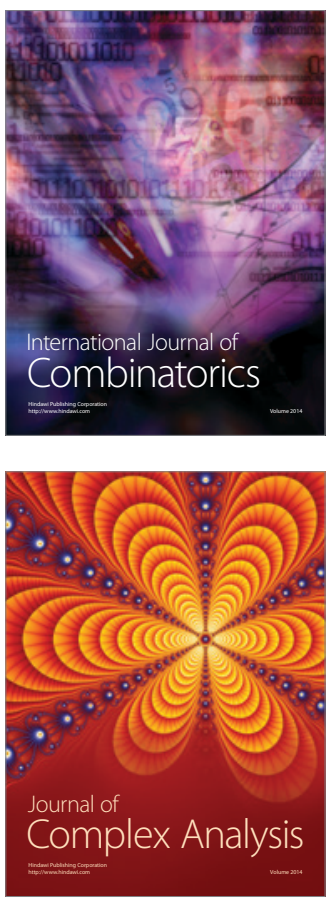

International Journal of

Mathematics and

Mathematical

Sciences
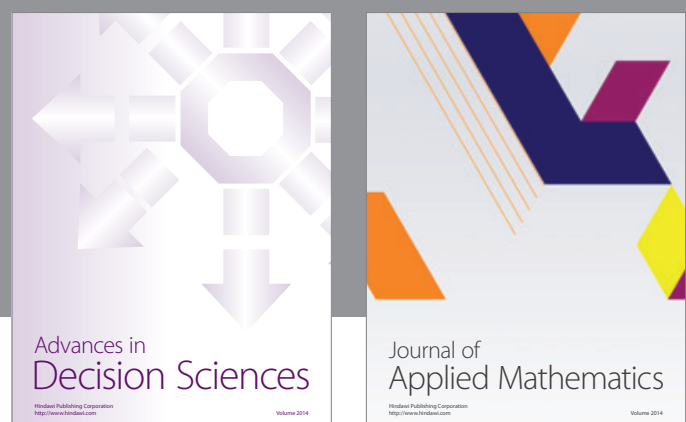

Journal of

Applied Mathematics
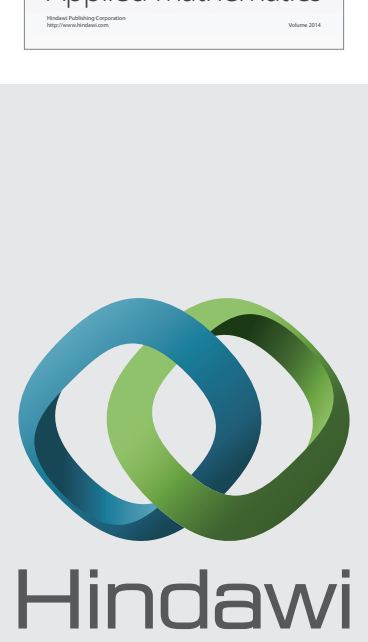

Submit your manuscripts at http://www.hindawi.com
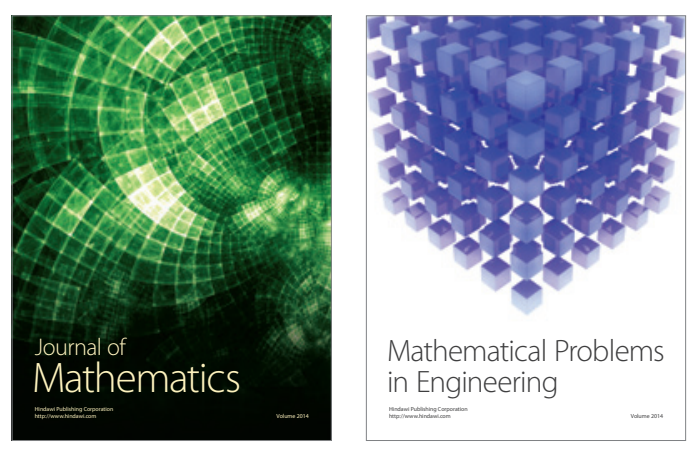

Mathematical Problems in Engineering
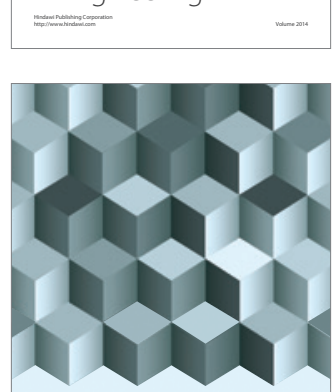

Journal of

Function Spaces
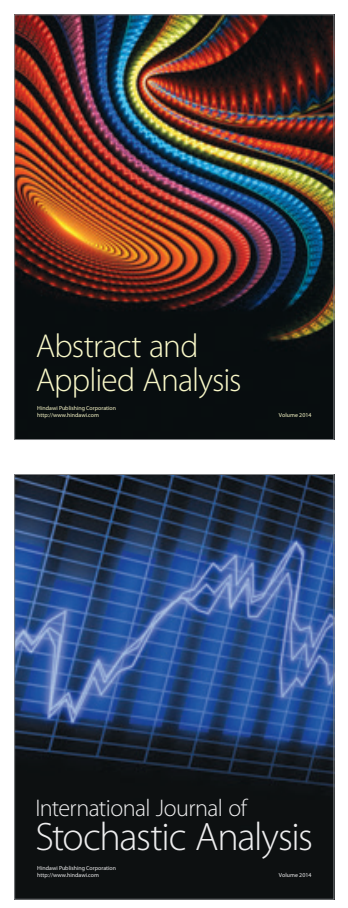

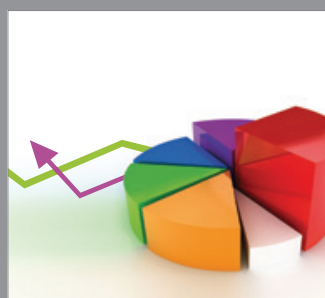

ournal of

Probability and Statistics

Promensencen
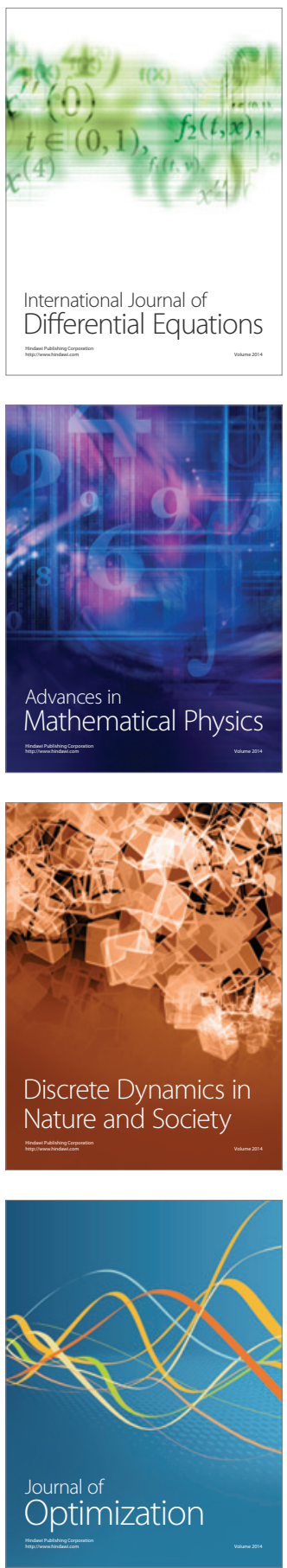